Revista de Psicología Vol. 31 (1), 2013 (ISSN 0254-9247)

\title{
Liderazgo, equipos y grupos de trabajo - su relación con la satisfacción laboral
}

\author{
Pablo Sebastián Pérez Vilar ${ }^{1}$, Susana Azzollini ${ }^{2}$ \\ Universidad Abierta Interamericana, \\ Consejo Nacional de Investigaciones Científicas y Técnicas, Argentina
}

\begin{abstract}
Se presenta los resultados obtenidos de una revisión bibliográfica de artículos científicos relacionados con la satisfacción laboral publicados entre los años 2000 y 2010. En el mismo se presentan las conclusiones de distintas investigaciones que señalan la relación entre el liderazgo, algunos aspectos de la supervisión, los grupos y equipos de trabajo, y su influencia sobre la Satisfacción Laboral. La literatura revisada señala principalmente una relación positiva entre el liderazgo transformacional y el modelo de supervisión SWA con la satisfacción laboral. También la importancia de la influencia de ciertos aspectos grupales como la eficacia, la cohesión grupal y la necesidad de compartir valores para que el trabajo grupal sea más satisfactorio que el trabajo individual.

Palabras clave: satisfacción laboral, liderazgo, grupos, trabajo.
\end{abstract}

\section{Leadership, teams, and collaborative groups - its relationship with job satisfaction}

Results obtained from a literature review of scientific articles related to job satisfaction published between the years 2000 and 2010, are presented. Findings of research studies analyzed indicate the relationship between leadership, some aspects of the supervision, groups, teams and its influence on job satisfaction. The revised bibliography noted mainly the positive relationship between transformational leadership and the SWA model of supervision on job satisfaction. Also the importance of the influence of certain aspects such as the efficacy group, group cohesion and the need to share values for group work be more satisfying than individual work.

Keywords: job satisfaction, leadership, workgroups, job.

1 Magister en Psicología Organizacional. Investigador del proyecto de investigación de la Maestría en Psicología Organizacional con Orientación Gerencial de la Universidad Abierta Interamericana. Dirección postal: Barrio Copello Edif. 17 - piso 9 - Dpto. "E", Ciudad Autónoma de Buenos Aires. Contacto: pablospv@hotmail.com

2 Doctora en Psicología de la UBA. Investigadora del Instituto de Investigaciones de la Facultad de Psicología. Investigadora Adjunta del Consejo Nacional de Investigaciones Científicas y Técnicas (CONICET). Directora de proyectos UBACyT No P046 y PIDDEF No 33/10 y del Proyecto de investigación de la Maestría en Psicología Organizacional con Orientación Gerencial de la Universidad Abierta Interamericana. Dirección postal: Fortunato Devoto 533, 6to piso, Ciudad Autónoma de Buenos Aires, Argentina. Contacto: susana1060@yahoo.com.ar 

La satisfacción laboral se ha convertido en los últimos años en un concepto popular en distintos campos de investigación. Esto se debe a que la Satisfacción laboral está relacionada con un gran número de variables de importancia en la vida de quienes son parte de una organización como: la productividad y el rendimiento, el estrés, el burnout, el ausentismo, la rotación entre otros (Luthans, (2002; Robbins, 1998; Spector 1997). Martínez Caraballo (2007) afirma que "el interés actual por la gestión de la Satisfacción laboral está alimentado por dos factores: el deseo de corregir la gestión empresarial del último periodo, enfocada unilateralmente a eficacia y flexibilidad, y querer responder a la nueva demanda del mercado laboral. Ambos factores llevan a concebir una nueva lógica en el trabajo" (Martínez Caraballo, 2007, p. 86). Por otra parte, un estudio internacional elaborado en el ańo 2001 por la consultora en gestión de recursos humanos "Hay Group" basado en entrevistas con trabajadores, área gerencial, profesionales y administrativos, reveló que el estilo del líder tiene un 70\% de impacto en la satisfacción de los empleados (Martínez Caraballo, 2007).

Sin embargo, Yukl (2002) plantea que existe cierta dificultad en encontrar causalidad o relaciones entre el liderazgo y la satisfacción laboral debido a la dificultad de explicar y medir todas las variables intervinientes. No obstante, la influencia de los jefes sobre los resultados organizacionales como la satisfacción con el trabajo ha demostrado ser significativa (Foong, 2001; Pezeshki Rad, Golshiri Esfahani \& Zamani Miandashti, 2008), pero esta relación "debe atender a las necesidades del momento y evaluar qué estilo/s es el más eficaz para esa situación en especial" (Cuadra Peralta \& Veloso Besio, 2007). También se ha investigado la relación líder-seguidor y su influencia sobre la satisfacción laboral (Golden \& Veiga, 2008) y se ha demostrado que la confianza de los seguidores hacia el líder se relaciona positivamente con dicha variable. 


\section{Metodología}

Para este estudio, se realizó una selección, descripción y análisis del corpus documental de trabajos de investigación realizados sobre satisfacción laboral publicados entre los años 2000 y 2010. Para ello, se procedió a una búsqueda exploratoria por internet en el buscador Google Académico (http://scholar.google. com.ar) y en la base de datos SciELO, utilizando como criterio de búsqueda las palabras "satisfacción laboral" además de los homónimos en inglés "job satisfaction" y "work satisfaction" en el título de trabajos publicados durante el periodo ya indicado. Se utilizó el mismo criterio en la búsqueda utilizando las bases de datos WilsonWeb, EbscoHost y OvidSP. La selección de los artículos, que forman parte de este trabajo, se hizo mediante la lectura de cada uno de los resúmenes de los mismos y la unidad de análisis estuvo constituida por cada uno de ellos que cumplió con los siguientes criterios:

Criterios de inclusión: Los límites establecidos en el proceso de búsqueda de los artículos se refieren únicamente al año de publicación (2000-2010) y a cada par de palabras de búsqueda (3 pares, 6 palabras). No se establecieron otros límites. Para proceder a la indagación de los artículos se escribió cada par de palabras de búsqueda y los resultados se filtraron según el período de publicación, obteniendo así los artículos completos que fueron descargados en el formato publicado o las referencias (autor, título, revista, año de publicación) y el resumen de cada una de las investigaciones, que fueron grabados en formato de texto. Los artículos seleccionados fueron aquellos que poseían en el título del artículo los pares de palabras mencionados y cuyos resultados analizaron u obtuvieron relaciones entre la Satisfacción laboral y otros constructos. Se incluyeron los trabajos publicados en Journals con referato tanto en forma virtual como en publicaciones impresas, escritos originalmente en (o traducidos al) inglés y/o castellano. En el caso de los constructos o instrumentos de medición que fueran referidos a 
bibliografía anterior al periodo mencionado, se recurrió a ellos con el objetivo de ampliar la información en relación con el artículo analizado.

Criterios de exclusión: Se excluyeron aquellos trabajos a los cuales no se pudo acceder a los textos completos por razones económicas o de acceso a las bases de datos consultadas. También se descartaron aquellos que no pertenecían específicamente al área de interés de la psicología organizacional, prefiriendo los divulgados en revistas con mayor factor de impacto y/o aquellos publicados con fecha más actual y con mayor descripción y referencias teóricas de los conceptos e instrumentos utilizados.

Criterios de eliminación: Se descartaron aquellos trabajos que solo evaluaban la Satisfacción laboral en una determinada población. Se eliminaron los artículos repetidos que se identificaron con diferentes palabras de búsqueda y los trabajos de replicación de resultados o que obtuvieron resultados similares utilizando la misma metodología y/o instrumento en el mismo tipo de población de otros trabajos ya escogidos. Una vez aplicados los distintos criterios la muestra quedo conformada por 388 trabajos del corpus inicial.

\section{Tipos de liderazgo y satisfacción laboral}

Existen cientos de trabajos (Yukl, 2002) que estudiaron la relación entre la satisfacción laboral y los estilos clásicos de liderazgo. Sin embargo, la mayoría no han sido concluyentes o han dado resultados inconsistentes (Adler \& Reid, 2008). Por ejemplo, el trabajo de Chen y Baron (2006) encontró que el estilo laissez-faire se relacionaba negativamente con la satisfacción con el trabajo, aunque ellos mismos escriben que los resultados obtenidos no alcanzan para explicar las variaciones del nivel de Satisfacción laboral. Algo similar ocurre con el trabajo de Sellgren, Ekvall y Tomson (2008) quienes encontraron relaciones entre la satisfacción laboral y los comportamientos de liderazgo, pero no encontraron diferencias entre aquellos orientados a la tarea y los 
orientados a las personas o a los cambios. El único hallazgo consistente es que los empleados se encuentran más satisfechos con su trabajo cuando el líder es moderadamente considerado (Yukl, 2002).

Es de considerar que en la mayoría de las investigaciones revisadas sobre liderazgo y satisfacción laboral se ha cambiado el enfoque del basado en las características del líder o la búsqueda de respuestas a la pregunta “¿cómo es el líder?” a otro basado en las conductas apropiadas para dirigir a los subordinados (Bass, 1981). Bass (1981) y Kouzes y Posner (1993) son los autores más citados, ya que han identificado conductas específicas que los líderes deben exhibir para influenciar a su gente y estas conductas pueden ser medidas y cuantificadas. Algunas aplicaciones de este enfoque, son las evaluaciones que se hacen sobre los programas de "mentoring", los cuales han demostrado mejorar la satisfacción laboral particularmente por su función modeladora del rol (Rhay-Hung et al. 2010; Selzer, 2008).

Las investigaciones revisadas muestran que los líderes que influyen en las actitudes positivas de los empleados (Villalba Moreno, 2001) y aquéllos que ejercen el liderazgo transformacional mejoran la satisfacción laboral de sus seguidores (Al Hussami, 2008; Cuadra Peralta \& Veloso Besio, 2007; Emery \& Barker, 2007 Judge, Piccolo \& Ilies, 2004; Shieh, Mills \& Waltz, 2001; Yi-Feng, 2009). Este tipo de líderes son aquéllos que cambian los valores básicos, creencias, y actitudes de los seguidores con el resultado final de inspirarlos para conseguir objetivos más altos y realizar el trabajo más allá de los niveles mínimos especificados por la organización (Bass, 1981).

El liderazgo transformacional es definido como un proceso comportamental comprendido por tres factores: carisma, estimulación intelectual y consideración individualizada de las necesidades de los seguidores (Bass \& Avolio, 1987), siendo la estimulación intelectual la que se correlaciona significativamente con la satisfacción laboral (Emery \& Barker, 2007) aunque los otros factores también han demostrado relaciones positivas con esta (Cuadra Peralta \& Veloso Besio, 2007). En este punto cabe señalar que este tipo de líderes poseen las siguientes características: a) son innovadores, se atreven a experimentar 
con nuevas y arriesgadas formas de hacer las cosas y alientan a otros a hacer lo mismo, b) son intuitivos, imaginan el futuro y describen sus ideas de manera vívida, involucrando a otros en ellas haciendo alusión a sus valores, intereses, esperanzas y sueños, c) despiertan confianza, comparten el poder, promueven el trabajo en equipo y la colaboración, lo cual ayuda a las personas a sentirse capaces y apoyadas d) son buenos ejemplos a seguir, en ellos no existe diferencia entre lo que predican y su actuación, e) apoyan a sus seguidores, animándolos, reconociendo y celebrando sus logros (Kouses \& Posner, 1993).

Los líderes que ejercen este tipo de liderazgo cuando retan el statu $q u o$, inspiran una visión compartida, capacitan y habilitan a los demás para actuar, sirven como modelo de actuación y animan a actuar, también promueven la satisfacción (Gaertner, 2000; Villalba Moreno, 2001). Por otra parte, Jaskyte (2003) encontró que la orientación a la producción, como componente del comportamiento del liderazgo transformacional se relaciona positivamente con la satisfacción laboral. Los estudiosos que investigan la relación entre estos dos constructos, también han descubierto que en ciertas situaciones el líder puede ser innecesario (Jernigan \& Beggs, 2010). Esta relación entre el liderazgo transformacional y la satisfacción puede verse moderada por la presencia de neutralizadores (como la orientación profesional) o sustitutos del liderazgo de gran magnitud, como la cohesión grupal, o las tareas intrínsecamente satisfactorias (Jernigan \& Beggs, 2010; Yusof \& Mohd Shah, 2008).

La teoría de los sustitutos del liderazgo señala que el liderazgo es efectivo en ausencia de sustitutos que actúen "en lugar de" el líder (Kerr \& Jermier, 1978). Los sustitutos son aquellas variables situacionales que debilitan el liderazgo y están relacionadas con el criterio analizado (la satisfacción laboral en este caso). En otras palabras la presencia del sustituto aumenta la satisfacción con el trabajo y debilita la influencia del líder. Mientras que los neutralizadores, como variable situacional, influyen en la respuesta de los subordinados en relación con el líder pero la variable en si no está relacionada con la satisfacción laboral, de esta forma un neutralizador vuelve al liderazgo transformacional 
ineficaz para incrementar la satisfacción en el trabajo (Yusof \& Mohd Shah, 2008).

Resulta importante destacar que también se han realizado investigaciones sobre la relación entre el modo de orientación regulatoria de las personas, la estrategia de influencia social y la satisfacción laboral. De estos estudios se concluye que la satisfacción de los empleados será más alta si las estrategias de influencia utilizadas por el líder encajan en (se ajustan con) el estilo regulatorio de los seguidores (Kruglanski, Pierro \& Higgins, 2007). Por ejemplo, los individuos con preocupaciones de locomoción fuertes quieren tomar medidas, comenzar rápido con las tareas, aún si esto significa no considerar todas las opciones, esta clase de individuos encajan con aquéllos que utilizan para influenciar estrategias de forzar (forcefull) cuya naturaleza es exigente, directiva y coercitiva pero manteniendo un tono neutro en lo que concierne a cuestiones políticas o evaluativas (liderazgo autocrático). Asimismo, se ha encontrado que las diferencias entre el estilo de liderazgo esperado y el que realmente se practica influye negativamente sobre la satisfacción laboral (Elpers \& Westhuis, 2008).

Otro modelo de liderazgo que ha sido investigado en relación con la satisfacción laboral, es la teoría LMX (Leader-Member Exchange) que se basa en la relación de intercambio social entre líder y seguidor o de los beneficios psicológicos obtenidos como confianza, apoyo, consideración y estima (Erdogan \& Enders, 2007; Schyns \& Croon, 2006; Stringer, 2006). La relación positiva entre LMX y la satisfacción laboral aumenta si se percibe apoyo organizacional (Erdogan \& Enders, 2007).

\section{Modelos de supervisión y satisfacción laboral}

Más allá del modelo de liderazgo elegido, varios trabajos han tratado la percepción de los empleados sobre el comportamiento del líder (Jaskyte, 2003), el de la dirección o del vínculo jerárquico y su relación con la satisfacción laboral (Robles Garcia et al., 2005); pero lo que mayormente se ha indagado al respecto es la influencia del liderazgo de los supervisores sobre la satisfacción con el trabajo de los empleados 
(Eklund \& Hallberg, 2000; Traut, Larsen \& Feimer, 2000). Las investigaciones han demostrado que la calidad de la relación entre supervisor y empleado está relacionada positivamente con la satisfacción con el trabajo (Garland \& McCarty, 2009; Hyrkas, Appelqvist-Schmidlechner \& Haataja, 2006; Stringer, 2006). Dentro de los modelos de supervisión relacionados específicamente con las tareas e investigado en relación con la satisfacción laboral, el modelo que destaca es el SWA. Este modelo se basa en tres características extraídas del trabajo: a) acuerdo mutuo con los objetivos de supervisión, b) tareas específicas relacionadas con la supervisión, c) el desarrollo de beneficios mediante bonos entre supervisor y supervisado; y cuando es visto de forma positiva, eleva la satisfacción laboral (Ramos-Sánchez et al., 2002).

\section{Trabajo en equipo y satisfacción laboral}

En las investigaciones revisadas solo se encontró un estudio que relaciona de forma estadísticamente significativa a la Satisfacción laboral con el trabajo en equipo en una institución gubernamental. Según estos autores (Newmark, Koehler \& Philippe, 2008) los grupos se hacen equipos cuando desarrollan un sentido del compromiso compartido y luchan por conseguir sinergia entre sus miembros. Contrario a la hipótesis de los autores, se comprobó que los empleados que trabajaban solos tenían niveles significativamente más altos de satisfacción que los que trabajaban en equipo. Estos autores justifican este resultado por la dificultad de las organizaciones para formar equipos de trabajo y sugieren que exista la probabilidad de que el trabajo en equipo produzca insatisfacción laboral. Otros (Wilson \& Crowe, 2008) encontraron que participar en un equipo es satisfactorio, pero si los miembros no comparten determinados valores la relación con la Satisfacción laboral se vuelve negativa. Aunque en algunos casos, como el de los trabajadores de instituciones de tratamientos a largo plazo, trabajar en un ambiente de equipo se relaciona positivamente con la Satisfacción laboral (Kinjerski \& Skrypnek, 2008; Moyle, Skinner, Rowe \& Gork, 2003). Mientras que el análisis realizado por Mohr y Zoghi (2008) en una 
organización de servicios de salud también arrojó resultados positivos. Por otra parte, los resultados obtenidos por McIntyre, Bartle, Landis y Dansby (2002) sugieren que la percepción de eficacia del grupo de trabajo también se relaciona positivamente con la Satisfacción laboral.

\section{Grupos de trabajo y satisfacción laboral}

En otro trabajo, Yi-Feng (2009) al estudiar los comportamientos de interacción de los grupos de trabajo, encontró relaciones positivas con la Satisfacción laboral, para este autor el grupo de trabajo es un grupo de tarea que comparte un propósito, objetivo, valor e interés común, donde los roles son interdependientes, y las destrezas complementarias. En otro estudio la interdependencia fue relacionada positivamente con la satisfacción laboral (Su-Fen \& Hsiao-Lan, 2005). Sin embargo, este estudio al igual que el de Yukl (2002), reconoce que este tipo de comportamientos por sí solos no afectan la Satisfacción laboral, sino que deben ser incorporados al liderazgo transformacional; aunque otros autores (Jernigan \& Beggs, 2010; Yusof \& Mohd Shah, 2008) demostraron que elementos como la cohesión grupal pueden reemplazar al líder en relación con la Satisfacción laboral, y Walsh, Matthews, Tuller, Parks y McDonald (2010) y Steinhardt, Dolbier, Gottlieb y McCalister, (2003) demostraron que la cohesión tiene una relación positiva significativa con la satisfacción con el trabajo. Según Roman (2001), las relaciones exitosas entre compañeros de trabajo están directamente asociadas con la Satisfacción laboral; y Zangaro y Soeken (2007) encontraron, al realizar un meta-análisis, que la colaboración médico-enfermero era la única variable cuya relación positiva con la Satisfacción laboral se mantenía homogénea a lo largo de los distintos estudios.

En otro estudio, Cox (2003) investigó la relación entre los conflictos intrapersonales, intragrupales e intergrupales encontrando que los conflictos intrapersonales e intragrupales se relacionaban negativamente con la satisfacción con el trabajo, mientras que los conflictos intergrupales no tenían relaciones significativas con el constructo. 
Sus resultados también indican que el conflicto intragrupal aumenta el efecto del conflicto intrapersonal sobre la satisfacción laboral. Esta autora encontró que el desempeño del equipo se relaciona positivamente con la satisfacción con el trabajo; siendo estos resultados congruentes con los obtenidos por Kunaviktikul, Nuntasupawat, Srisuphan y Booth (2000) sobre que los empleados que experimentan altos niveles de conflicto, tienen bajos niveles de satisfacción laboral. También se ha demostrado que el estilo de integración el cual, según Rahim and Bonoma (en Tabak \& Orit, 2007), involucra altos niveles de preocupación por el interés propio y el de la otra parte al momento de la resolución de conflictos interpersonales, es el que más se relaciona con la satisfacción con el trabajo (Tabak \& Orit, 2007).

Una técnica grupal que ha demostrado relacionarse con la Satisfacción laboral, es la de los grupos Balint, en las reuniones de estos grupo los miembros describen espontáneamente los encuentros con los pacientes y el grupo discute sobre estos encuentros, enfocándose en la historia y los sentimientos que despierta, para facilitar nuevas maneras de comprender la relación médico - paciente (Johnson, Nease, Milberg $\&$ Addison, 2004). En este sentido, un estudio descriptivo-cualitativo Dorte e Inger (2008) revelaron que la participación en estos grupos incrementaba la satisfacción, aunque reconocen que este método no es aplicable a todos los empleados.

\section{Discusión}

La revisión bibliográfica realizada muestra que las investigaciones recientes han cambiado el eje de análisis de los estilos de liderazgo al centrar su atención en aquellas acciones que realiza el líder que logra sus objetivos y al mismo tiempo genera satisfacción laboral en sus seguidores. Estas investigaciones han encontrado relaciones entre el ajuste de la estrategia de liderazgo y los estilos regulatorios de los seguidores, particularmente si el líder inspira confianza y ejerce un estilo de liderazgo transformacional, destacando la importancia de este tipo de 
liderazgo en el comportamiento de los grupos de trabajo. Es por todo esto, y considerando que existen pocas investigaciones realizadas en América Latina que resulta necesario evaluar las estrategias de dirección y de resolución de conflictos que se utilizan en nuestras organizaciones. Este artículo ofrece algunas referencias para la revisión y análisis de los comportamientos dentro de las organizaciones, pues vincula dos de las principales dinámicas de la organización, la relación líder-seguidor y los grupos de trabajo con la satisfacción laboral. Por ejemplo, en las investigaciones revisadas también se encontró que tareas intrínsecamente muy satisfactorias pueden quitarle importancia a la influencia del liderazgo sobre la satisfacción laboral. Incluso se ha demostrado que factores como la eficacia y la cohesión grupal tienen tanto valor en las organizaciones de la actualidad que incluso pueden opacar la influencia del factor liderazgo en la satisfacción laboral (Jernigan \& Beggs, 2010; Yusof \& Mohd Shah, 2008)

Por otra parte, más allá del liderazgo ejercido, en los trabajos resalta la influencia significativa de la buena relación entre jefes, mentores y supervisores con los empleados (incluyendo el análisis de teorías específicas como la LMX o el método SWA); lo que resulta llamativo, ya que a diferencia del liderazgo transaccional — que se guía por la premisa de que hay subordinados al líder, que los esfuerzos de estos son premiados por el líder (Stringer, 2006) y que quienes ejercen este tipo liderazgo suelen caracterizarse por ser carismáticos (Yammarino \& Dubinsky, 1994) — teorías como la LMX puede aplicarse a cualquier estilo de liderazgo (Stringer, 2006) y relacionarse positivamente con la satisfacción laboral. Es por ello que se considera que este trabajo presenta la relación entre algunos constructos que resultaría interesante profundizar en nuestros países, dado la falta de trabajos empíricos relacionados con la temática especifica. 


\section{Referencias}

Adler R. \& Reid J. (2008). The effects of leadership styles and budget participation on job satisfaction and job performance. Asia-Pacific Management Accounting Journal, 3 (1), 21-46. Recuperado el 8 de enero de 2011 de http://web.ebscohost.com Al Hussami, M. (2008). A Study of Nurses' Job Satisfaction: The Relationship to Organizational Commitment, Perceived Organizational Support, Transactional Leadership, Transformational Leadership, and Level of Education. European Journal of Scientific Research 22 (2), 286-295 Recuperado el 29 de noviembre de 2010 de http://www.eurojournals.com/ejsr_22_2_14.pdf

Bass, B. M. (1981). Stogdill's Handbook of Leadership. Nueva York: The Free Press.

Bass, B., Avolio B. \& Goodheim L. (1987). Biography and the assessment of transformational leadership at the world class level. Journal of Management, 13, 7-19. Recuperado el 8 de enero de 2011 de http://web.ebscohost.com

Chen, H. \& Baron, M. (2006). Nursing directors' leadership styles and faculty members' job satisfaction in Taiwan. The Journal of Nursing Education, 45(10), 404-411. Recuperado el 8 de enero de 2011 de http://web.ebscohost.com

Cox, K. (2003). The effects of intrapersonal, intragroup, and intergroup conflict on team performance effectiveness and work satisfaction. Nursing Administration Quarterly, 27(2), 153-163. Recuperado el 29 de noviembre de 2010 de http://www.emeraldinsight.com

CuadraPeralta,A.\&VelosoBesio,C.(2007).Liderazgo, ClimaySatisfacción laboral en las Organizaciones. Universum [online]. 22(2) Recuperado el 4 de diciembre de 2010 de http://www.scielo.cl/scielo. php?script=sci_arttext\&pid=S0718-23762007000200004\&lng =es\&nrm=iso

Dorte, K. \& Inger, H. (2008). Balint Groups as a Means to Increase Job Satisfaction and Prevent Burnout Among General Practitioners. 
Annals of Family Medicine, 6(2), 138-145. Recuperado el 08 de enero de 2011 de http://web.ebscohost.com

Eklund, M. \& Hallberg, I. (2000). Factors influencing job satisfaction among Swedish occupational therapists in psychiatric care. Scandinavian Journal of Caring Sciences, 14, 162-171. Recuperado el 4 de diciembre de 2010 de http://web.ebscohost.com

Elpers, K. \& Westhuis, D. (2008). Organizational leadership and its impact on social workers' job satisfaction: a national study. Administration in Social Work, 32(3), 26-43. Recuperado el 8 de enero de 2011 de http://web.ebscohost.com

Emery, C. \& Barker, K. (2007). The effect of transactional and transformational leadership styles on the organizational commitment and job satisfaction of customer contact personnel. Journal of Organizational Culture, Communications \& Conflict, 11(1), 77-90. Recuperado el 8 de enero de 2011 de http://web.ebscohost.com

Erdogan, B. \& Enders, J. (2007). Support From the Top: Supervisors' Perceived Organizational Support as a Moderator of Leader-Member Exchange to Satisfaction and Performance Relationships. Journal of Applied Psychology, 92 (2), 321-330. Recuperado el 6 de diciembre de 2010 de http://www.ovid.com Foong, J. (2001). Leadership behaviours: effects on job satisfaction, productivity and organizacional commitment. Journal of Nursing Management, 9, 191-204. Recuperado el 8 de enero de 2011 http://web.ebscohost.com

Gaertner, S. (2000). Structural determinants of job satisfaction and organizational commitment in turnover models, Human Resource Management Review, 9, 479-493. Recuperado el 8 de enero de 2011 de http://web.ebscohost.com

Garland, B. \& McCarty, W. (2009). Job satisfaction behind walls and fences: A study of prison health care staff. Criminal Justice Policy Review, 20, 188-208. Recuperado el 29 de noviembre de 2010 de http://cjp.sagepub.com/content/early/2009/03/12/0887403 409333261.full.pdf+html 
Golden, T. \& Veiga, J. (2008). The impact of superior-subordinate relationships on the commitment, job satisfaction, and performance of virtual workers. Leadership Quarterly, 19(1) 77-88. Recuperado el 4 de diciembre de 2010 de http://www.sciencedirect.com

Hyrkas, K., Appelqvist-Schmidlechner, K. y Haataja, R. (2006). Efficacy of clinical supervision: influence on job satisfaction, burnout and quality of care. Journal of Advanced Nursing, 55(4), 521-535. Recuperado el 4 de enero de 2011 de http://web. ebscohost.com

Jaskyte, K. (2003). Assessing Changes in Employees' Perceptions of Leadership Behavior, Job Design, and Organizational Arrangements and Their Job Satisfaction and Commitment. Administration in Social Work, 27(4), 25-39. Recuperado el 2 de diciembre de 2010 de http://web.ebscohost.com

Jernigan, E. \& Beggs, J. (2010). Substitutes for leadership and job satisfaction: is there a relationship? Journal of Organizational Culture, Communications \& Conflict, 14(2), 97-106. Recuperado el 2 de diciembre de 2010 de http://web.ebscohost.com

Johnson, A., Nease D., Milberg L. \& Addison R. (2004). Essential characteristics of effective Balint group leadership. Fam Med. 36(4), 253-259. http://www.stfm.org/fmhub/fm2004/April/ Alan253.pdf

Judge, T., Piccolo, R. \& Ilies, R. (2004). The forgotten ones, The Validity of Consideration and Iniciating Structure in Leadership Research. Journal of Applied Psychology, 89(1), 36-51. Recuperado el 6 de diciembre de 2010 de http://www.ovid.com

Kinjerski, V. \& Skrypnek, B. (2008). The promise of spirit at work: increasing job satisfaction and organizational commitment and reducing turnover and absenteeism in long-term care. Journal of Gerontological Nursing, 34(10), 17-25. Recuperado el 8 de enero de 2011 de http://web.ebscohost.com

Kouzes, M. \& Posner, Z. (1993). El Desafío del Liderazgo. Granica, Buenos Aires. 
Kruglanski, A., Pierro, A. \& Higgins, E. (2007). Regulatory Mode and Preferred Leadership Styles: How Fit Increases Job Satisfaction. Basic \& Applied Social Psychology, 29(2), 137-149. Recuperado el 8 de enero de 2011 de http://web.ebscohost.com

Kunaviktikul, W., Nuntasupawat, R., Srisuphan, W. \& Booth, R. (2000). Relationships among conflict, conflict management, job satisfaction, intent to stay, and turnover of professional nurses in Thailand. Nursing \& Health Sciences, 2(1), 9-16. Recuperado el 8 de enero de 2011 de http://web.ebscohost.com

Luthans, F. (2002). Comportamiento Organizacional. México: McGrawHill Interamericana Editores S.A

Martínez Caraballo, N. (2007). Recursos humanos y management empresarial. El caso de Satisfacción laboral. Cuadernos de Ciencias Económicas y Empresariales, (1er semestre) (52). Recuperado el 2 de diciembre de 2010 de http://dialnet.unirioja.es/servlet/ dcart?info $=$ link\&codigo $=2692060 \&$ orden $=163608$

McIntyre, R., Bartle, S., Landis, D. \& Dansby, M. (2002). The effects of equal opportunity fairness attitudes on job satisfaction, organizational commitment, and perceived work group efficacy. Military Psychology, 14, 299-319. Recuperado el 29 de noviembre de 2010 de http://web.ebscohost.com

Mohr, R. \& Zoghi, C. (2008). High-Involvement Work Design And Job Satisfaction. Industrial \& Labor Relations Review, 61(3), 275-296. Recuperado el 29 de noviembre de 2010 de http:// web.ebscohost.com

Moyle, W., Skinner, J., Rowe, G. \& Gork, C. (2003). Views of job satisfaction and dissatisfaction in Australian long-term care. Journal of Clinical Nursing, 12(2), 168-176. Recuperado el 8 de enero de 2011 de http://web.ebscohost.com

Newmark, L., Koehler, J. \& Philippe, T. (2008). Effects of work teams on job satisfaction. International Journal of Business Research, 8(2), 228-234. Recuperado el 8 de enero de 2011 de http://web. ebscohost.com 
Pezeshki Rad, G., Golshiri Esfahani, Z. \& Zamani Miandashti, N. (2008). Investigation of Leadership Style Correlates Affecting Jihad-e-Keshavarzi Staffs Job Satisfaction in Yazd. Journal Agric. Science Technology, 10, 421-429. Recuperado el 29 de noviembre de 2010 de http://mohitpardaz.com/mp/images/stories/article1. pdf

Ramos-Sánchez, L., Esnil, E., Goodwin, A., Riggs, S., Touster, L., Wright, L. et al. (2002). Negative supervisory events: Effects on supervision satisfaction and supervisory alliance. Professional Psychology: Research and Practice, 33, 197-202. Recuperado el 29 de noviembre de 2010 de http://www.uiowa.edu/ - counspsy/faculty/ SUPERVISION\%20COURSE/NEGATIVE/NEGATIVE.pdf

Rhay-Hung, W., Ching-Yuan, H., Wen-Chen, T., Li-Yu, C., Syr-En, L. \& Mei-Ying, L. (2010). Exploring the impact of mentoring functions on job satisfaction and organizational commitment of new staff nurses. BMC Health Services Research, 10240-248. Recuperado el 8 de enero de 2011 de http://web.ebscohost.com Robbins, S. (1998) Comportamiento organizacional. 7a Edición. México: Editorial Prentice Hall.

Roman, M. (2001). From AMSN. Teams, teammates, and team building. MEDSURG Nursing, 10(4), 161-162. Recuperado el 8 de enero de 2011 de http://web.ebscohost.com

Schyns, B. \& Croon, M. (2006). A model of task demands, social structure, and leader-member exchange and their relationship to job satisfaction. International Journal of Human Resource Management 17 (4), 602-615. Recuperado el 29 de noviembre de 2010 de http://web.ebscohost.com

Sellgren, S., Ekvall, G. \& Tomson, G. (2008). Leadership behaviour of nurse managers in relation to job satisfaction and work climate. Journal of Nursing Management, 16(5), 578-587. Recuperado el 8 de enero de 2011 de http://web.ebscohost.com

Selzer, E. (2008). Effectiveness of a Seminary's Training and Mentoring Program and Subsequent Job Satisfaction of its Graduates. 
Journal of Research on Christian Education, 17(1), 25-53. Recuperado el 8 de enero de 2011 de http://web.ebscohost.com Shieh, H., Mills, M.\& Waltz, C. (2001). Academic leadership style predictors for nursing faculty job satisfaction in Taiwan. Journal of Nursing Education, 40, 203-209. Recuperado el 29 de noviembre de 2010 de http://www.ncbi.nlm.nih.gov/pubmed/11355758 Spector, P. (1997). Job Satisfaction: Application, Assessment, Cause and Consequences. Nueva York: Harper \& Row.

Steinhardt, M., Dolbier, C., Gottlieb, N. \& McCalister, K. (2003). The relationship between hardiness, supervisor support, group cohesion and job stress as predictors of job satisfaction. American Journal Health Promotion 17(6), 382-389. Recuperado el 8 de enero de 2011 de http://web.ebscohost.com

Stringer, L. (2006). The Link Between the Quality of the Supervisor-Employee Relationship and the Level of the Employee's Job Satisfaction. Public Organization Review, 6(2), 125-142. Recuperado el 29 de noviembre de 2010 de http://web.ebscohost. com

Su-Fen, C. \& Hsiao-Lan, C. (2005). Relationship between Job Characteristics and Organizational Citizenship Behavior: The mediational role of Job Satisfaction. Social Behavior \& Personality: An International Journal, 33(6), 523-539. Recuperado el 29 de noviembre de 2010 de http://web.ebscohost.com

Tabak, N. \& Orit, K. (2007). Relationship between how nurses resolve their conflicts with doctors, their stress and job satisfaction. Journal of Nursing Management, 15(3), 321-331. Recuperado el 29 de noviembre de 2010 de http://web.ebscohost.com

Traut, C., Larsen, R. \& Feimer, S. (2000). Hanging on or Fading out? Job Satisfaction and the Long-term Worker. Public Personnel Management, 29 (3), 343-351. Recuperado el 29 de noviembre de 2010 de http://eric.ed.gov/ERICWebPortal/search/detailmini.jsp?_nfpb=true\&_\&ERICExtSearch_SearchValue_0=EJ615 987\&ERICExtSearch_SearchType_0=no\&accno=EJ615987 
Villalba Moreno, O. (2001). Incremento de la satisfacción y del compromiso organizacional de los empleados a través del liderazgo efectivo. Revista Latinoamericana de Administración, 26, 5-17.

Walsh, B., Matthews, R., Tuller, M., Parks, K. \& McDonald, D. (2010). A Multilevel Model of the Effects of Equal Opportunity Climate on Job Satisfaction in the Military. Journal of Occupational Health Psychology, 15(2), 191-207. Recuperado el 6 de diciembre de 2010 de http://www.ovid.com

Wilson, B. \& Crowe, M. (2008). Maintaining equilibrium: a theory of job satisfaction for community mental health nurses. Journal of Psychiatric \& Mental Health Nursing, 15(10), 816-822. Recuperado el 8 de enero de 2011 de http://web.ebscohost.com

Yammarino, F. \& Dubinsky, A. (1994). Transformational Leadership Theory: Using Levels Of Analysis To Determine Boundary Conditions. Personnel Psychology, 47(4), 787-811. Recuperado el 4 de diciembre de 2010 de http://web.ebscohost.com

Yi-Feng, Y. (2009). An investigation of group interaction functioning stimulated by transformational leadership on employee intrinsic and extrinsic job satisfaction: An extension of the resource-based theory perspective. Social Behavior \& Personality: An International Journal, 37(9), 1259-1277. Recuperado el 8 de enero de 2011 de http://web.ebscohost.com

Yukl, G. (2002). Leadership in Organizations, $5^{\text {ta }}$ Ed. Upper Saddle River, NJ: Prentice Hall.

Yusof, A. \& Mohd Shah, P. (2008). Transformational Leadership and Leadership Substitutes in Sports: Implications on Coaches' Job Satisfaction. International Bulletin of Business Administration. EuroJournals. Recuperado el 8 de enero de 2011 de http://www. eurojournals.com/IBBA_3_03.pdf

Zangaro, G. \& Soeken, K. (2007). A meta-analysis of studies of nurses' job satisfaction. Research in Nursing \& Health, 30(4), 445-458. Recuperado el 4 de diciembre de 2010 de http://web.ebscohost.com

Recibido el 12 de enero, 2013 Aceptado el 3 de mayo, 2013 